\title{
Transcatheter Embolization of a Large Symptomatic Pelvic Arteriovenous Malformation with Glubran 2 Acrylic Glue
}

\author{
R. Gandini · G. Angelopoulos $\cdot$ D. Konda $\cdot$ M. Messina $\cdot$ M. Chiocchi \\ T. Perretta $\cdot$ G. Simonetti
}

Received: 3 May 2007/ Accepted: 8 October 2007

(C) Springer Science+Business Media, LLC 2007

\begin{abstract}
A young patient affected by a pelvic arteriovenous malformation (pAVM) with recurrent episodes of hematuria following exercise, underwent transcatheter embolization using Glubran 2 acrylic glue (GEM, Viareggio, Italy). All branches of the pAVM were successfully occluded. The patient showed prompt resolution of symptoms and persistent occlusion of the pAVM at the 6 month follow-up.
\end{abstract}

Keywords Acrylic glue - Embolization - Glubran 2 .

Liquid embolic agent $\cdot$ Pelvic arteriovenous malformation

\section{Introduction}

Pelvic arteriovenous malformations (pAVMs) are rare congenital lesions and present a difficult diagnostic and therapeutic challenge [1]. Therapy is aimed at the complete closure of the arteriovenous communications using endovascular techniques or traditional surgery. Surgical resection of pAVMs is associated with a high failure and recurrence rate [2]. Transcatheter embolization has been suggested as the primary therapeutic modality or as a presurgical intervention to reduce bleeding and maximize successful resection $[2,3]$.

R. Gandini · G. Angelopoulos ( $₫)$ - D. Konda - M. Messina M. Chiocchi - T. Perretta - G. Simonetti

Interventional Radiology Department, University Hospital of Rome "Tor Vergata", Rome, Italy

e-mail: giorginos78@msn.com

\section{Case Report}

A 31-year-old man (an amateur athlete) with no significant medical history was referred to our department because of recurrent episodes of macroscopic hematuria after exercise (playing soccer). Urine analysis performed between episodes of macrohematuria detected the presence of microhematuria. There was no history of previous trauma or surgical treatment in the pelvic region. Physical examination was negative. There was no audible bruit, visible or palpable pulsations, or hyperemic overlying skin. The patient experienced no pain.

Cystoscopy performed 7 days previously in another institution revealed no pathologic findings. Pelvic ultrasonography revealed a mass attached to the left posterior part of the urinary bladder. A subsequent volumetric computed tomography angiography (angio-VCT) of the pelvis detected a pAVM with feeding branches originating from the left hypogastric artery. The nidus of the pAVM involved the urinary bladder and the distal part of the left ureter. After consultation with the vascular surgeon, due to the complexity of the pAVM's arterial supply and the high risk of hemorrhage, endovascular embolization of the pAVM was decided on. Informed consent was obtained from the patient prior to the procedure.

The right common femoral artery was punctured using the Seldinger technique and a $5 \mathrm{Fr}$ introducer sheath (Radiofocus, Terumo, Japan) was positioned. The left internal iliac artery was then catheterized using a 5 Fr "Simmons 1" angiographic catheter (Glidewire, Terumo, Japan). Angiography confirmed the presence of a large pAVM with multiple feeding arteries originating from the anterior branches of the left hypogastric artery (Fig. 1A). The large nidus of the pAVM drained into the left hypogastric vein through varicose veins. A microcatheter (SP, Terumo, 

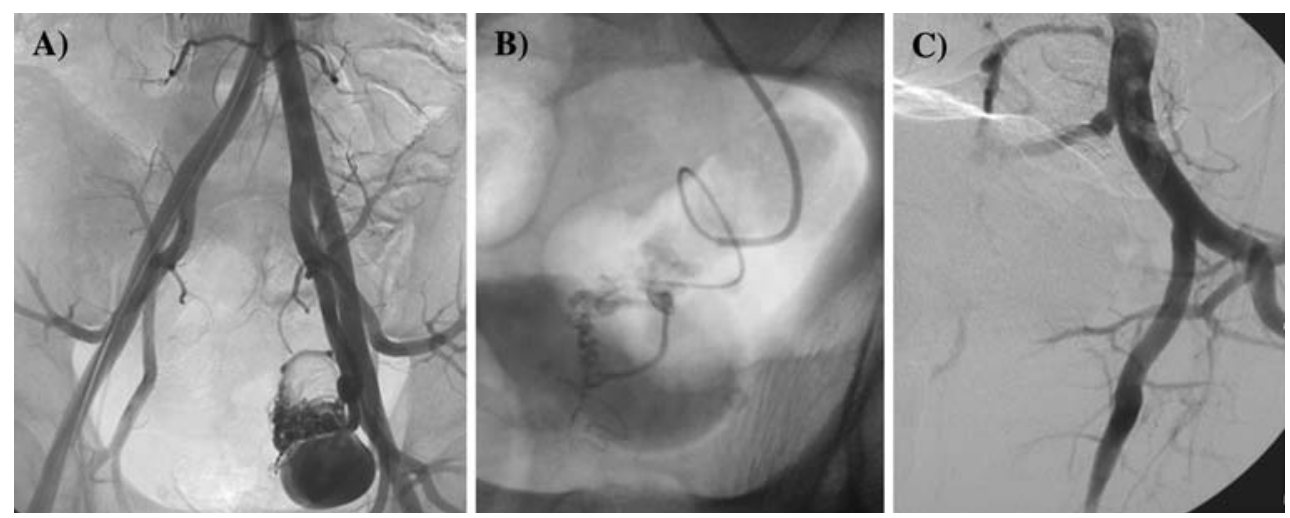

Fig. 1 (A) Pelvic arteriogram showing the pAVM, which is fed by multiple branches of the left hypogastric artery (anterior branches). An aneurysm can be also observed before the emptying of the pAVM into the ipsilateral vein. (B) Embolization of the feeding branches

Japan) was introduced through the angiographic catheter and a major feeding branch of the nidus was superselectively catheterized. A small amount of contrast agent was then injected to confirm the optimal position of the microcatheter adjacent to the nidus and to assess the volume of acrylic glue required for embolization. The nidus was then embolized by injecting $1 \mathrm{ml}$ of Glubran 2 acrylic glue (GEM, Viareggio, Italy), mixed with $2.5 \mathrm{ml}$ of lipiodol (1:2.5 ratio) to enable its fluoroscopic visualization (Fig. 1B). The same procedure was repeated until all the feeding branches were embolized.

Digital subtraction angiography (DSA) performed immediately after the procedure (Fig. 1C) and angio-VCT $24 \mathrm{hr}$ later confirmed the complete devascularization and thrombosis of the nidus.

Follow-up performed by urine analysis 1,3 , and 6 months after the procedure revealed the absence of macroscopic and microscopic hematuria. Angio-VCT performed 6 months after the procedure confirmed the persistent devascularization of the pAVM with no signs of bladder and/or ureter ischemia (Fig. 2).

To our knowledge this is the first case report in the literature of a pAVM treated by transcatheter embolization using Glubran 2 acrylic glue (GEM, Viareggio, Italy).

\section{Discussion}

Pelvic AVMs are uncommon vascular lesions formed by multiple abnormal communications between the arterial and venous system (without a normal capillary network). Especially in males, only a few cases have been previously described in literature [4]. The etiology has been a subject of controversy, but it is generally accepted that these lesions are congenital or acquired after trauma or surgery. The natural history is variable. They can remain with Glubran 2 and lipiodol after selective catheterization using a microcatheter (SP, Terumo). (C) Post-embolization arteriogram shows the occlusion of all embolized branches with complete devascularization of the nidus

asymptomatic for years and, at times, indefinitely. When symptoms develop, there may be pain, mass effect (invasion-compression of adjacent organs), hemorrhage, highoutput heart failure, and impotence [3].

The management of congenital pelvic vascular malformations has been a difficult problem for decades. Surgical treatment alone has historically been inadequate and has often led to damage to adjacent structures with high recurrence rates [5]. Most authors agree that simple ligation of feeding arteries has no value, since new collaterals develop rapidly [6]. Superselective catheterization of feeding vessels and the transcatheter administration of embolic agents have been shown to be effective methods for managing these lesions. Jacobowitz et al.. have published the largest study to date from a single institution regarding pelvic lesions. They propose that transcatheter embolization should be the initial therapy [3].

The development of flexible small-caliber catheters has allowed superselective cannulation of branch vessels and angiographic delineation of the anatomy of vascular malformations. Feeding vessels can be identified, and transcatheter embolization therapy can then be performed with the goal of obliterating the nidus. The range of available embolization materials (Gelfoam, PVA foam particles, Ivalon pledgets, and cyanoacrylate adhesives such as NBCA) has evolved in recent years. Detachable coils and balloons provide proximal occlusion but, as they cannot penetrate the nidus, their role is limited. Polyvinyl alcohol (PVA) particles may be useful in some cases, but determining the optimal size to use is difficult and their use may be associated with pulmonary embolism. Another concern with PVA particles may be recanalization or development of arterial collaterals. Liquid agents may be the most suitable because of their ability to form a cast of the vasculature that needs to be occluded [3]. Acrylic glues, once deposited into the nidus, provide permanent occlusion and prevent its 

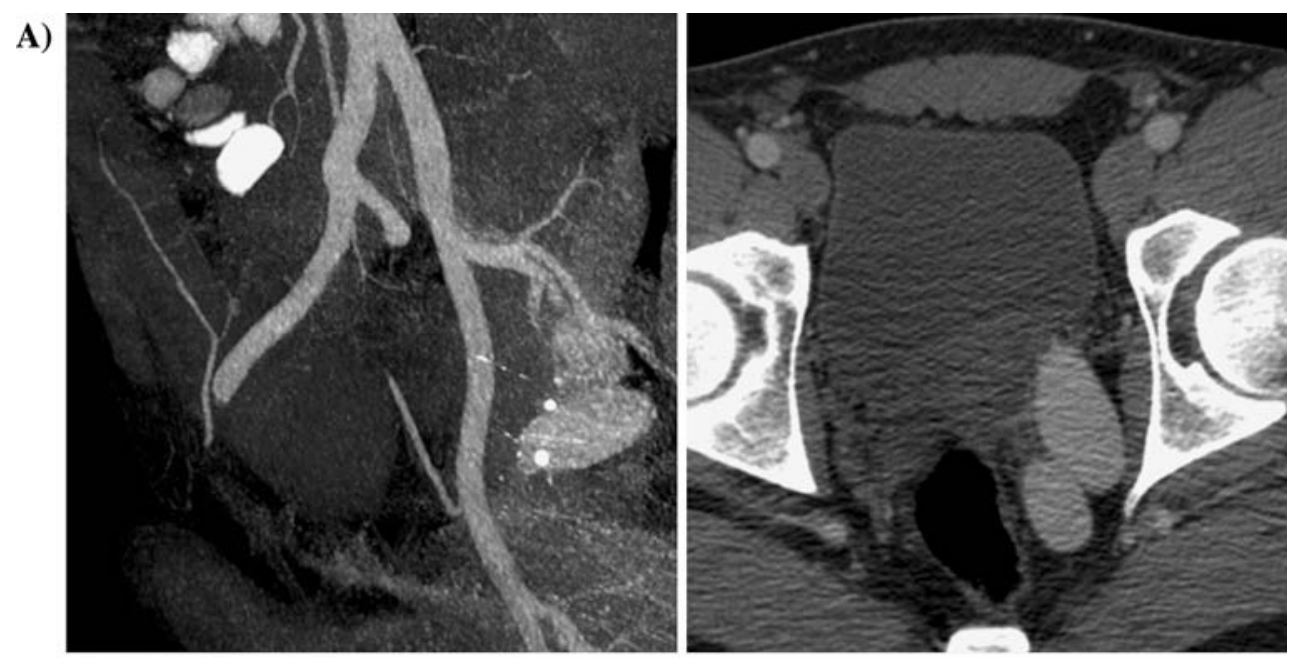

B)
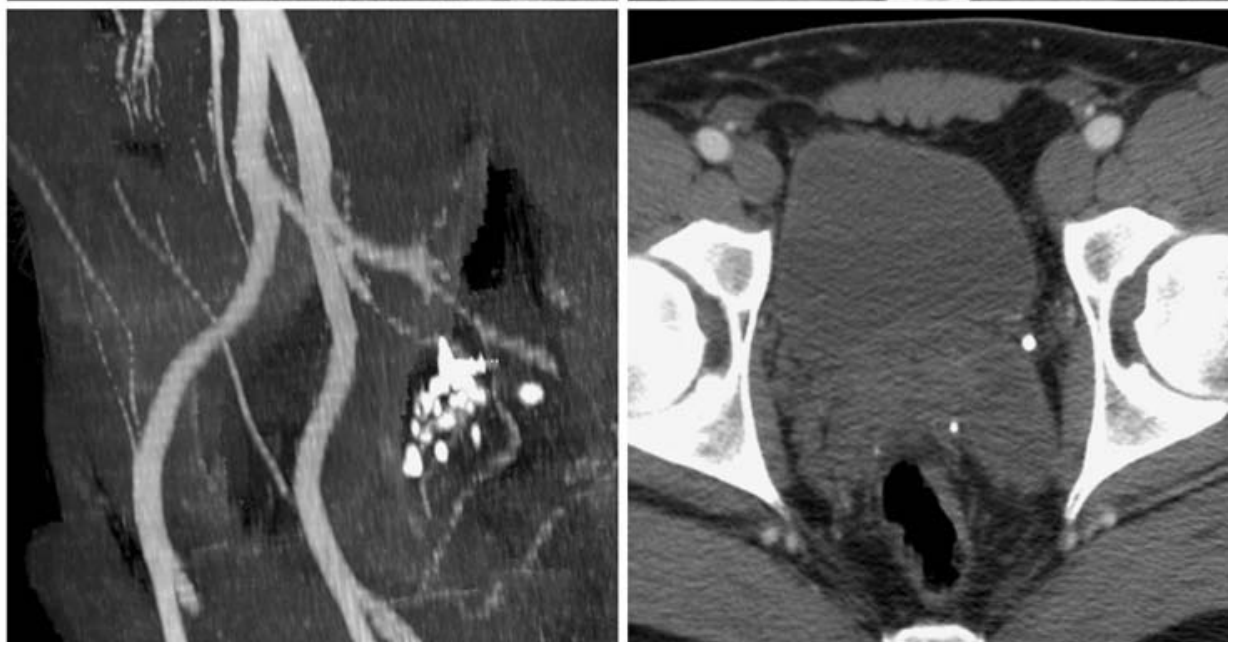

Fig. 2 (A) The preoperative angio-VCT demonstrates a large pAVM involving the urinary bladder and the distal part of the left ureter. (B) The postembolization angio-VCT shows the complete devascularization of the pAVM

replenishment through feeding branches. Although recurrence of the PAVM is possible, due to recanalization and/or neovascular recruitment, the embolization procedure can be repeated if symptoms recur [3]. Adjunctive surgical therapy should be reserved for those patients whose symptoms do not improve with embolization [3].

$\mathrm{N}$-butyl-Cyanoacrylate (NBCA) is a monomer acrylic glue which polymerizes on contact with blood, and subsequently causes a permanent occlusion. To avoid adherence to the surrounding tissue of the thin catheters required for the superselective embolization, NBCA has to be injected through a catheter washed with a 5\% dextrose solution, and the catheter has to be withdrawn promptly after injection [7].

Glubran 2 acrylic glue (GEM, Viareggio, Italy) has been used in the treatment of cerebral vascular anomalies in an experimental study without major complications [10], and we have had experience using this embolic agent in patients with post-traumatic priapism [9]. We therefore chose Glubran 2 as the embolic agent. Being a liquid, this embolizing agent is also able to conform to the shape of the tortuous arteries supplying AVMs.

Glubran 2 is an acrylic glue authorized for use in surgical and endovascular procedures. The glue needs to be mixed with lipiodol before use to enable its fluoroscopic visualization. The co-monomer of Glubran 2 is comprised of a monomer of NBCA and a monomer of MS (owned by GEM). MS allows the monomer of NBCA to polymerize with a smaller exothermic reaction $\left(45^{\circ} \mathrm{C}\right)$ and a slightly longer polymerization time [8].

Compared with the monomer NBCA, Glubran 2 causes less pain to the patient and is associated with a lower risk of adherence of the catheter to the tissue, allowing a greater ease of use. Another advantage of Glubran 2 is its relatively low cost. Other liquid embolic agents described in literature which can offer an alternative are absolute alcohol and ethylene vinyl alcohol (Onyx) [9, 10]. A major concern with absolute alcohol is its cardiac effect and, in agreement with Jacobowitz et al. [3], we believe that absolute alcohol should be reserved for venous 
malformations, where low flow allows the alcohol to remain at the injection site. On the other hand, although the safety and efficacy of Onyx has previously been described in literature [10], its high cost is a major disadvantage. Additionally the injection of Onyx is painful and moderate sedation or general anesthesia may be necessary in order to perform more effective embolization.

Endovascular therapy with embolic agents has become an increasingly useful treatment option. We believe that in expert hands, primary embolization using acrylic glues such as Glubran 2 is safe, feasible, and very efficient. Since the long-term safety and effectiveness of any liquid embolic agent has not been established yet, more studies with larger patient populations are needed in order to confirm our results. Glubran 2 acrylic glue should be considered a valid alternative in the treatment of pAVMs.

\section{References}

1. Trout HH, McAllister HA, Giordano JM, Rich NM (1985) Vascular malformations. Surgery 97:36-41
2. Flye MW, Jordan BP, Schwartz MZ (1983) Management of congenital arteriovenous malformations. Surgery 94:740-747

3. Jacobowitz G, Rosen R, Rockman C, et al. (2001) Transcatheter embolization of complex pelvic vascular malformations: Results and long-term follow-up. J Vasc Surg 33(1):51-55

4. Game X, et al. (2002) Congenital pelvic arteriovenous malformation in male patients: A rare cause of urological symptoms and role of embolization. Eur Urol 42:407-412

5. Gomes MM, Bernatz PE (1970) Arteriovenous fistulas: A review and 10 year experience at the Mayo Clinic. Mayo Clin Proc 45:81-102

6. Pritchard DA, Maloney JD, Bernatz PE, Symmonds RE (1978) Surgical treatment of congenital pelvic arteriovenous malformation. Mayo Clin Proc 53:607-611

7. Gandini R, Spinelli A, Konda D, Reale CA, Fabiano S, Pipitone V, Simonetti G (2004).Superselective embolization in posttraumatic priapism with Glubran 2 acrylic glue. Cardiovasc Intervent Radiol 27:544-548

8. Leonardi M, Barbara C, Simonetti L, Giardino R (2002) Glubran 2: A new acrylic glue for neuroradiological endovascular use: Experimental study on animals. Intervent Neororadiol 8:245-250

9. Yakes W, Krauth L, Ecklund J, et al. (1997) Ethanol endovascular management of brain arteriovenous malformations: Initial results. Neurosurgery 40:1145-1154

10. Castaneda F, Goodwin SC, et al. (2002) Treatment of pelvic arteriovenous malformations with ethylene vinyl alcohol copolymer (Onyx). J Vasc Interv Radiol 13:513-516 